J. Clin. Chem. Clin. Biochem.

Vol. 16, 1978, 397-402

\title{
Quantitative Bestimmung von Serum-Proteinen mit einem neuen Laser-Nephelometer
}

\author{
Von B. Baruth, M. J. Caputo, H. Deicher und H. Leyssens
}

Abteilung für klinische Immunologie und Transfusionsmedizin, Department Innere Medizin, Medizinische Hochschule Hannover, Bundesrepublik Deutschland, und S. A. Travenol Laboratories N. V., Lessines, Belgien

(Eingegangen am 25. Oktober 1977/13. März 1978)

\begin{abstract}
Zusammenfassung: Diese Arbeit berichtet über methodische Aspekte quantitativer Serum-Proteinbestimmungen mittels eines neuen Laser Nephelometers (PDQ ${ }^{\mathrm{TM}}$-Laser-Nephelometer der Firma Hyland). Die Präzision der Methode in der Serie ergab Variationskoeffizienten zwischen $2,5 \%$ und $4,6 \%$ bei den vier gemessenen Serumproteinen (IgG, IgA, IgM und (3). Die Präzision von Tag zu Tag ergab einen durchschnittlichen Variationskoeffizienten von $8 \%$ mit einem niedrigsten Wert von 5\%. An insgesamt 60 Probanden ermittelte Normalwerte zeigten vergleichbare Ergebnisse mit der radialen Immundiffusionstechnik. Auch in pathologischen Bereichen wurden vergleichbare Ergebnisse erzielt. Die Methode ist damit als verläßlich anzusehen und bietet gegenüber der radialen Immundiffusion nicht nur den Vorteil einer weit höheren Präzision, sondern auch die Möglichkeit, Testbefunde innerhalb von 2 Stunden herauszugeben.
\end{abstract}

\section{Quantitative determination of serum proteins using a new laser nephelometer}

Summary: Methodological aspects of quantitative serum protein determinations, using a newly developed laser nephelometer (PDQ ${ }^{\mathrm{TM}}$ laser nephelometer, Hyland), are reported. For within-day precision, determination of the four serum proteins IgG, IgA, IgM and C'3, showed coefficients of variation between $2.5 \%$ and $4.6 \%$. For precision from day to day, the average variation coefficent was $8 \%$, the lowest value being $5 \%$. Provisional normal values for the four proteins were ascertained from a small group of 60 healthy individuals. These results, as well as those obtained in states of disease, correlate with values obtained by radial immunodiffusion. It is concluded that the method is reliable, and has a number of important advantages compared to radial immunodiffusion; namely, the much higher precision, better sensitivity, particularly in lower concentration ranges, and availability of results within two hours.

\section{Einleitung}

Obwohl radiale Immunodiffusion $(1,2,3)$ und RocketElektroimmunodiffusion (4) zur routinemäßigen Bestimmung einzelner Serumproteine Eingang in viele diagnostische Laboratorien gefunden haben, können einige Nachteile dieser Methoden wie eingeschränkter Testbereich, schlechte Reproduzierbarkeit, lange Inkubationszeiten und das Fehlen jeglicher Mechanisierung nicht übersehen werden. Zur mechanisierten quantitativen Bestimmung von Proteinen mittels spezifischer Antiseren werden seit einigen Jahren nephelometrische Verfahren benutzt $(5,6)$. Bei der Nephelometrie unterscheidet man zwei Prinzipien:

1. die klasșische Nephelometrie, die das in einem Winkel von $90^{\circ}$ abgestreute Licht verwendet, und

2. Verfahren, bei denen das in Vorwärtsrichtung in einem unterhalb von $90^{\circ}$ liegenden Winkel abgestreute Licht gemessen wird. Wird ein Laserstrahl ${ }^{1}$ ) als Licht:

1) Laser ist ein Acronym für Light Amplifịcation by Stimulated Emission of Radiation. quelle verwandt, so ist diese Technik wesentlich empfindlicher als die klassische Nephelometrie.

Letztere Meßanordnung ließ erwarten, daß besonders im unteren Empfindlichkeitsbereich der mit nephelometrischen Verfahren erfaßbaren Immunpräzipitation bessere Ergebnisse erzielt werden konnten. Die Ergebnisse der Untersuchung zeigen, daß ein auf dieser Basis konstruiertes Gerät für die quantitative Bestimmung von einzelnen Proteinen in biologischen Flüssigkeiten mit Hilfe präzipitierender spezifischer Antiseren gut geeignet ist und hinsichtlich Präzision und Empfindlichkeit anderen Meßverfahren deutlich überlegen erscheint.

\section{Material und Methoden}

1. Gerät, Reagenzien, Seren

Alle Messungen wurden mit dem $\mathrm{PDQ}^{\mathrm{TM}}$-Laser-Nephelometer (Protein Discrete Quantitation/Transversal Mode Laser-Nephelometer, Hyland Division of Travenol Laboratories Inc., Costa Mesa, California, USA.) durchgefuhrt. 


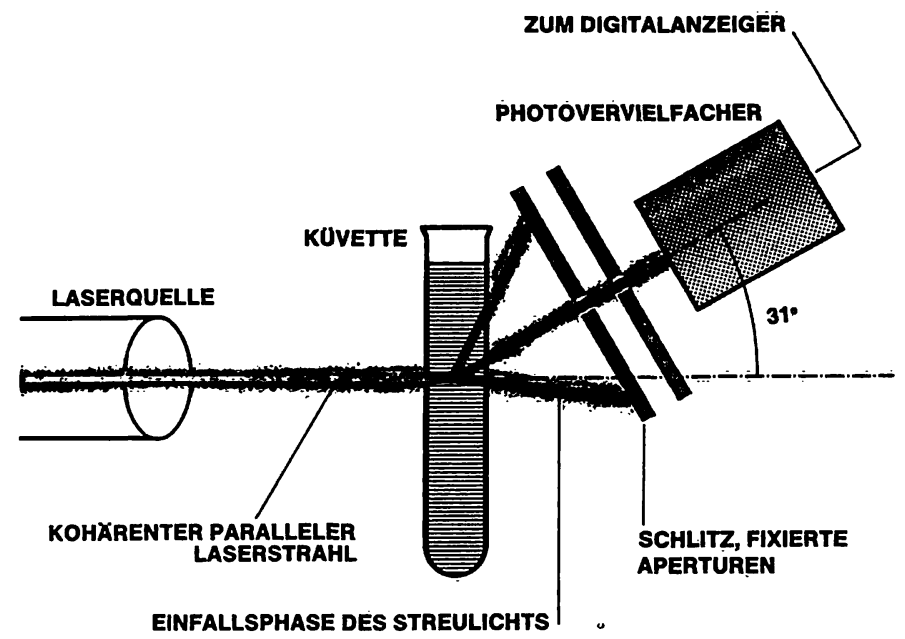

Abb. 1. Schematische Meßanordnung im PDQTM-Laser-Nephelometer.

Der He-Ne-Laserstrahl dieses Instruments liegt im sichtbaren Rotbereich des Spektrums und emittiert eine Wellenlänge von $632,8 \mathrm{~nm}$. Speichervorrichtungen und ein kleiner Computer erlauben die Speicherung von Leerwerten (Serum-, Antikörper-, Lösungsmittelleerwert), die automatisch von der Endablesung subtrahiert werden. Der Meßwinkel von $31,8^{\circ}$ (Abb. 1) wurde aufgrund ausgedehnter experimenteller Untersuchungen mit Immunkomplexen unter Berücksichtigung der Benutzung einfacher Rundküvetten gewählt. Dieser Winkel erlaubt eine optimale Messung der durch Antigen-Antikörper-Komplexe hervorgerufenen Lichtstreuung. Immersionsflüssigkeiten (z. B. Toluol), die für den Brechungsindex der Glasküvette kompensieren sollen, finden keine Anwendung. Die Ergebnisse werden in „relativer Lichtstreuung“ angegeben und sind am Digitalanzeiger des Gerätes abzulesen. Die ,relative“ Lichtstreuung ist ein relatives Maß für die durch die spezifischen AntigenAntikörper-Komplexe entstandene Zunahme der Lichtstreuung in der Meßküvette, wobei zur Erzielung maximaler Empfindlichkeit für jedes gemessene Protein der Meßwert (mV) eines Referenzserums mit bekannter (hoher) Konzentration des betreffenden Proteins $=180$ gesetzt wird. Alle anderen Konzentrationen werden dann relativ zu diesem Meßpunkt angegeben. Die absoluten Konzentrationen im $\mathrm{g} / \mathrm{l}$ ergeben sich aus den jeweiligen Standardkurven.

Verdünnte monospezifische Antiseren, Pufferlösung, Referenzseren, Einmalküvetten und Kontrollproben wurden von der Firma Hyland Division, Travenol Laboratories Inc., Costa Mesa, California, USA, geliefert. Zur Filtration der Reagenzien wurden Swinnex ${ }^{2}$ )-Filtrationsvorsätze ( $\phi 47 \mathrm{~mm}$ ) und -Membranfilter $(0,45 \mu \mathrm{m})$ verwendet. Zum Abmessen der Reagenzien und Proben wurden Oxford-Pipetten ${ }^{3}$ ) mit einstellbaren Volumina $\left(25,50\right.$ und $100 \mu$ l) sowie Brand-Dispensetten $\left.{ }^{4}\right)(5 \mathrm{ml}$ Volumen) benutzt.

\section{Methodenbeschreibung}

Referenz- und Patientenseren wurden 1:100 mit filtrierter $0,15 \mathrm{~mol} / 1$ Natriumchlorid-Lösung verdünnt. Bei jeder Bestimmung müssen Serumleerwert und Antikörperleerwert neben der Testprobe mitgefürt werden. In allen untersuchten Seren wurden IgG, IgA, IgM und C3 quantitativ bestimmt. Für die IgG- und IgA-Bestimmungen wurden $25 \mu \mathrm{l}$, für die IgM- und C3-Bestimmungen $100 \mu \mathrm{l}$ der Serumproben in zwei Küvetten pipettiert.

$\left.{ }^{2}\right)$ Millipore GmbH, Neu-Isenburg, Bundesrepublik Deutschland.

3) Oxford Laboratories $\mathrm{GmbH}$, München, Bundesrepublik Deutschland.

4) Rudolf Brand, Wertheim, Bundesrepublik Deutschland.
Zum Serumleerwert wurde $1,0 \mathrm{ml}$ filtrierte $0,15 \mathrm{~mol} / 1$ Natriumchlorid-Lösung, zur Testprobe $1,0 \mathrm{ml}$ des zu gleịchen Teilen mit Puffer verdünnten Antiserums hinzugefügt. Ein separat angesetzter Antikörperleerwert enthielt $1 \mathrm{ml}$ verdünntes Antiserum und 25 bzw. $100 \mu 10,15 \mathrm{~mol} / 1$ Natriumchlorid-Lösung.

Alle Küvetten wurden $1 \mathrm{~h}$ bei Raumtemperatur inkubiert. Diese Zeit ist jedoch nicht kritisch, und die Ablesung kann zu jedem Zeitpunkt zwischen 1-4 h erfolgen.

Das Instrument wird zunächst elektronisch auf 0 eingestellt und der Serumleerwert gemessen. Danach wird der Testbereich eingestellt und der Antikörperleerwert gemessen. Anschließend an diese Schritte erfolgt die Ablesung der Testproben. Die auf dem Digitalanzeiger erscheinenden Ergebnisse stellen die relative Lichtstreuung der nach der Inkubation in der Testprobe gebildeten spezifischen Immunkomplexe dar. Anhand dieser Werte können dann auf den Referenzkurven die Serumwerte in $\mathrm{g} / \mathrm{l}$ spezifischen Proteins ermittelt werden.

\section{Erstellung der Referenzkurven}

Referenzkurven für die verschiedenen Serumproteine wurden mit Hilfe von delipidierten Standard-Humanseren, deren Gehalte an IgG, IgA, IgM und C3 mittels radialer Immundiffusion ermittelt worden waren, erstellt (7). Für jede Kurve wurden sechs verschiedene Proteinkonzentrationen doppelt bestimmt. Anschließend wurde auf linearem Millimeterpapier Konzentration gegen ,relative Lichtstreuung “ aufgetragen. Typische Referenzkurven für IgA und IgM sind in den Abbildungen 2 und 3 dargestellt.

\section{Radiale Immundiffusion}

Zur Durchführung der quantitativen Proteinbestimmungen mittels radialer Immundiffusion wurden Immunoplate IIIPlatten (Hyland Division, Travenol GmbH, München) in der üblichen Methodik benutzt (siehe auch 1.).

\section{Ergebnisse}

Bei der Untersuchung dieses Instruments wurden die Präzision der Meßmethode, der Methodenvergleich mit der radialen Immundiffusion und die Ermittlung der Normalbereiche fự die verschiedenen Proteine besonders berücksichtigt. 


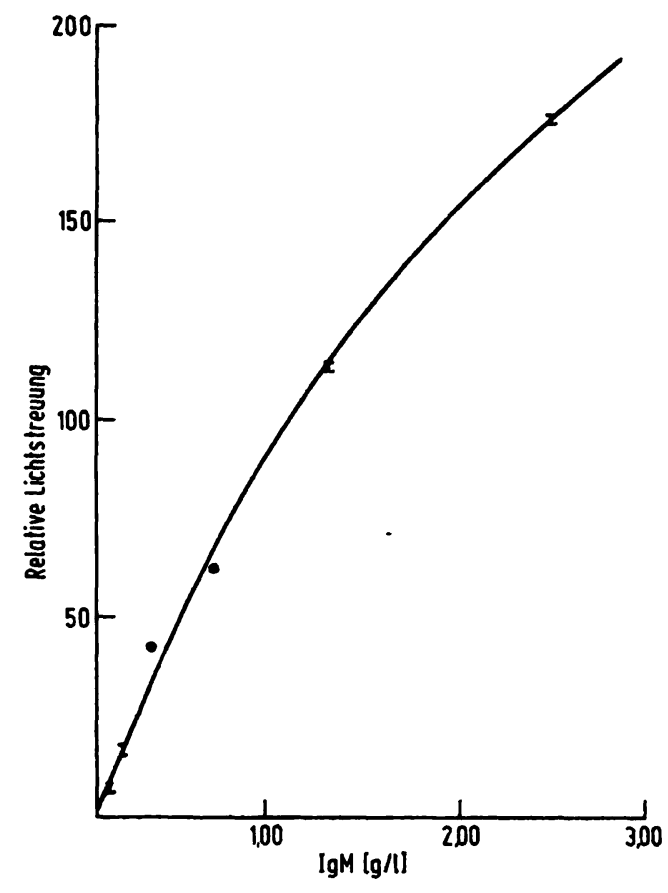

Abb. 2. Standardkurve für IgM (Doppelbestimmungen aus Standard-Humanserum, Verdünnung 1:100).

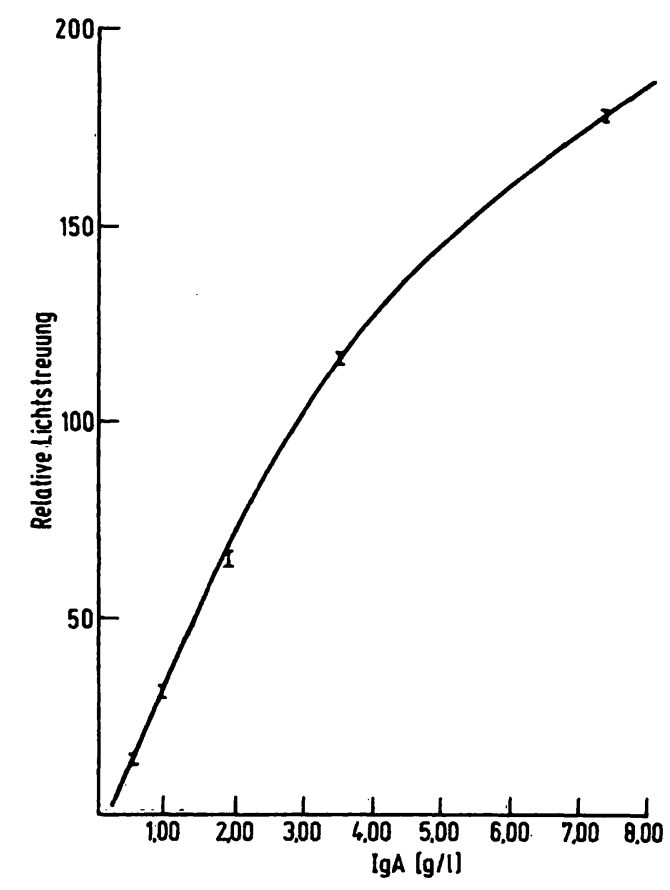

Abb. 3. Standardkurve für IgA (Doppelbestimmungen aus Standard-Humanserum, Verdünnung 1:100). über einen Zeitraum von 4 Monaten durchgeführt. Die Konzentrationen der 5 Kontrollseren (Tab. 1) wurden so gewählt, daß die Präzision für jedes spezifische Antikörpersystem weitgehend über den gesamten Testbereich einer Referenzkurve ermittelt werden konnte.

In Tabelle 2 sind die Ergebnisse der Präzision in der Serie als mittlere Variationskoeffizienten (VK) und Variationskoeffizientbereiche für alle Konzentrationen dargestellt. Tabelle 3 zeigt das gleiche für die Präzision von Tag zu Tag.

Aus den in Tabelle 2 dargestellten Daten ergibt sich für die Präzision in der Serie ein durchschnittlicher Variationskoeffizient von $4 \%$. Ähnlich verhält es sich mit der Präzision von Tag zu Tag (Tab. 3), bei der ein durchschnittlicher Variationskoeffizient von $8 \%$ erreicht wurde; der niedrigste Wert lag bei $5 \%$.

Die in Abbildung 4 zusammengefaßten Daten zeigen, daß die bei dieser Technik erreichte Reproduzierbarkeit kaum abhängig von der Konzentration des zu messenden Proteins ist. Niedrige und hohe Proteinkonzentrationen können so mit gleich guter Genauigkeit gemessen werden. Dieses Ergebnis steht in deutlichem Gegensatz zur radialen Immundiffusion, wo man bei niedrigen Proteinkonzentrationen Variationskoeffizienten von über $20 \%$, im allgemeinen jedoch regelmäßig über $15 \%$ erhält $(8,9,10)$.

Tab. 1. Konzentrationen (g/l) der Kontrollseren (Blindproben), die zur Ermittlung der Präzision dienten.

\begin{tabular}{|c|c|c|c|c|c|c|}
\hline \multirow{2}{*}{$\begin{array}{l}\text { Anti- } \\
\text { körper- } \\
\text { Spezi- } \\
\text { fität }\end{array}$} & \multicolumn{5}{|c|}{ Kontroll-Serum } & \multirow{2}{*}{$\begin{array}{l}\text { Referenzkurv } \\
\text { (Bereich g/l) }\end{array}$} \\
\hline & 1 & 2 & 3 & 4 & 5 & \\
\hline IgG & 16,25 & 7,95 & 2,80 & 1,40 & 21,67 & $0,80-27,00$ \\
\hline IgA & 4,58 & 2,22 & 0,78 & 0,39 & 6,11 & $0,24-7,40$ \\
\hline IgM & 2,26 & 1,15 & 0,37 & 0,19 & 3,01 & $0,07-2,35$ \\
\hline C3 & 2,68 & 1,40 & 0,44 & 0,22 & 3,57 & $0,09-3,25$ \\
\hline
\end{tabular}

Táb. 2. Präzision in der Serie. Mittlere Variationskoeffizienten (VK) und Variationskoeffizientenbereiche $(n=20)$.

\begin{tabular}{lllll}
\hline & IgG & IgA & IgM & C3 \\
\hline VK (\%) & 3,4 & 3 & 4,6 & 2,5 \\
Bereich & $0,5-4,8$ & $0,9-4,5$ & $2,1-7,1$ & $0,5-5,3$ \\
\hline
\end{tabular}

Tab. 3. Präzision in der Serie. Mittlere Variationskoeffizienten (VK) und Variationskoeffizientenbereiche $(n=20)$. dierte humane Kontrollseren in 5 verschiedenen Konzentrationen $20 \mathrm{X}$ innerhalb einer Testserie eingesetzt. Diese Blindproben wurden vom Hersteller zur Verfügung gestellt. Für die Präzision von Tag zu Tag wurden an. den gleichen Kontrollseren 55 Doppelbestimmungen

\begin{tabular}{lllll}
\hline & IgG & IgA & IgM & C3 \\
\hline VK (\%) & 10,6 & 8,8 & 7,9 & 5,3 \\
Bereich & $4,4-18,4$ & $5,0-14,9$ & $4,6-11,1$ & $3,7-7,3$ \\
\hline
\end{tabular}




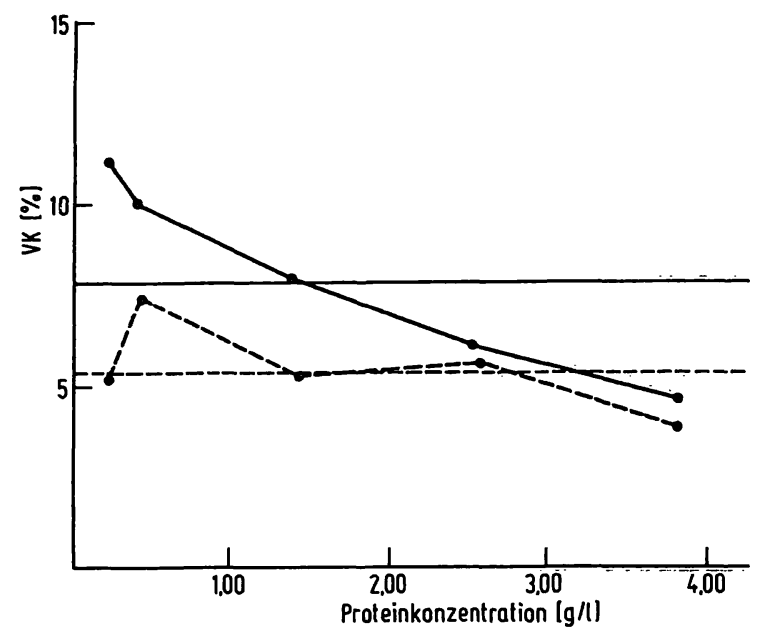

Abb. 4. Darstellung der ermittelten Variationskoeffizienten (VK) für IgM und C3 bei verschiedenen Proteinkonzentrationen, ermittelt an 1:100-verdünnten StandardHumanseren.

$\bullet-\bullet$ IgM $\bullet---\bullet C 3$

Die Geraden geben den jeweils mittleren VK an.

Methodenvergleich mit der radialen Immunodiffusion

Um die Brauchbarkeit dieser Technik für klinische Zwecke zu erproben, wurde sie mit der weitverbreiteten radialen Immunodiffusionstechnik verglichen. Insgesamt wurden 90 Serumproben gesunder und kranker Personen sowohl am Nephelometer als auch mit der radialen Immunodiffusion für jedes spezifische Antigen gemessen. Die von den 2 Methoden erhaltenen Daten wurden verglichen und mittels einer Regressionsgleichung ausgewertet. Die Konzentrationen der Serumproben lagen für:

IgG zwischen $2,40-45,00 \mathrm{~g} / 1$

IgA zwischen $0,40-20,00 \mathrm{~g} / 1$

IgM zwischen $0,10-50,00 \mathrm{~g} / 1$

C3 zwischen $0,10-3,50 \mathrm{~g} / 1$

Alle Daten sind in Tabelle 4 zusammengefaßt.
Die in Tabelle 4 vorliegenden Daten zeigen zwischen den zwei verschiedenen Methoden nur geringfügige Unterschiede; erkennbar wird dies aus den fast identischen Mittelwerten für jedes spezifisches Protein. Die Regressionsgleichung und die Korrelationskoeffizienten zeigen eine ausgezeichnete Ǔbereinstimmung zwischen den beiden Methoden. Lediglich bei einigen Seren von Patienten mit paraproteinämischen Hämoblastosen stellten wir zwischen der Nephelometrie und der radialen Immunodiffusion erhebliche Unterschiede fest.

\section{Normalwerte}

In unserer Studie wurden an einem kleinen Kollektiv von insgesamt 60 Serumproben gesunder Erwachsener Normalwerte ermittelt. Diese Normalwerte erheben keinen Anspruch auf Gültigkeit und stellen lediglich ein Testkollektiv dar, das die Vergleichbarkeit der Methode mit anderen demonstrieren soll. Das Kollektiv -setzte sich aus Seren von je 30 weiblichen und männlichen Personen zusammen, deren Alter zwischen 18 und 60 Jahren lag. Unterschiede hinsichtlich Rasse, Körpergewicht, Tageszeit oder Nüchternzustand wurden nicht berücksichtigt. Bei der Auswertung der Normalwerte wurde insbesondere auf Unterschiede zwischen männlichen und weiblichen Personen geachtet. Die für IgG, IgA und Komplement C3 erhaltenen Daten lassen weder eine bedeutsame Verschiebung der Normalverteilung noch einen Konzentrationsunterschied aufgrund des Geschlechts erkennen. Nur bei der Analyse des IgM ergab sich eine erhebliche Verschiebung der Normalverteilung; diese konnte durch Verwendung der logarithmierten Werte korrigiert werden. Außerdem fanden sich Unterschiede zwischen Werten männlicher und weiblicher Testpersonen. Aus diesem Grunde wurden die für IgM erhaltenen Daten für männliche und weibliche Personen separat aufgefuihrt. Die in Tabelle 5 aufgeführten Daten zeigen die erhaltenen Mittelwerte $(x)$ und die in \pm 2 s angegebenen Vertrauensbereiche.

Tab. 4. Methodenvergleichsstatistik für IgG, IgA, IgM und C3.

\begin{tabular}{|c|c|c|c|c|c|}
\hline $\begin{array}{l}\text { Spezifisches } \\
\text { Protein }\end{array}$ & $\mathrm{n}$ & Methode & $\begin{array}{l}\text { Mittelwert } \\
\text { g/1 }\end{array}$ & Regressionsgleichung & $\begin{array}{l}\text { Korrelations- } \\
\text { koeffizienten }\end{array}$ \\
\hline \multirow[t]{2}{*}{ IgG } & 90 & Nephelometer & 11,33 & $y=28,8+0,99(x)$ & 0,995 \\
\hline & & radiale Immunodiffusion & 11,17 & & \\
\hline \multirow[t]{2}{*}{$\operatorname{Ig} A$, } & 85 & Nephelometer & 3,33 & $y=21,7+0,96(x)$ & 0,996 \\
\hline & & radiale Immunodiffusion & 3,24 & & \\
\hline \multirow[t]{2}{*}{ IgM } & 84 & Nephelometer & 5,87 & $y=4,3+0,99(x)$ & 0,993 \\
\hline & & radiale Immunodiffusion & 5,85 。 & & . \\
\hline \multirow[t]{2}{*}{ C3 } & 93 & Nephelometer & 1,18 & $y=0,90+1,04(x)$ & $0,98 \overline{8}$ \\
\hline & & radiale Immunodiffusion & 1,12 & & \\
\hline
\end{tabular}


Tab. 5. Vorläufige Normalwerte aus einem Kollektiv von 60 Gesunden.

\begin{tabular}{lll}
\hline Protein & $\begin{array}{l}\text { g/1 } \\
\text { Mittelwert }(\overline{\mathbf{x}})\end{array}$ & $\begin{array}{l}\text { Vertrauensbereich } \\
( \pm 2 \mathrm{~s})\end{array}$ \\
\hline IgG & 9,62 & $\begin{array}{c}6,32- \\
12,92\end{array}$ \\
& & $1,02-$ \\
IgA & 2,54 & 4,06 \\
& & $0,82-$ \\
C3 & 1,17 & 1,67 \\
& & $0,56-$ \\
IgM (ठ) & 1,14 & 2,30 \\
& & $0,64-$ \\
IgM (\$) & 1,23 & 2,34 \\
\hline
\end{tabular}

\section{Diskussion}

Das PDQ ${ }^{\mathrm{TM}}$ Laser-Nephelometer hat sich für quantitative Bestimmungen spezifischer Proteine als gut geeignet erwiesen. Wie die mittleren VK für Abweichungen von Tag zu Tag wie für die Serie zeigen, ist die Präzision dieser Methode wesentlich besser als diejenige von radialer Immunodiffusion. Außerdem ist die Präzision unabhängig von der Konzentration des gemessenen Proteins, was für die radiale Immunodiffusion nicht zutrifft. Ein besonderer Vorteil des Nephelometers ist der weite Konzentrationsbereich der Referenzkurve, so daß Proben mit niedrigen und hohen Konzentrationen in einem Testdurchgang bestimmt werden können. Da Seren für die Untersuchung je nach Bestimmungsmethode bis $100 \mathrm{fach}$ verdünnt werden, können mit diesem Gerät verläßliche quantitative Bestimmungen nicht nur im niedrigen Konzentrationsbereich, so z. B. für Immunglobuline bei Immundefekten verschiedener Art, sondern auch in anderen Körperflüssigkeiten, wie z. B. Liquor cerebrospinalis, vorgenommen werden (10). Für niedrigere Konzentrationsbereiche benötigte man lediglich eine spezielle Referenzkurve, die mit geeigneten Referenzpräparaten leicht erstellt werden kann. Ein weiterer Vorteil der Methode ist deren Schnelligkeit, Testbefunde können schon innerhalb von 2 Stunden herausgegeben werden, was besonders gegenüber der vielfach benutzten radialen Immunodiffusion ins Gewicht fällt. Die quantitative Bestimmung einzelner Serumproben kann so zeitgerecht in das diagnostische Mosaik des klinischen Laboratoriums am Krankenbett eingebaut werden.
Es wurde schon darauf hingewiesen, daß bei Seren von Patienten mit paraproteinämischen Hämoblastosen, z. B. Myelomen, keine verläßlichen Werte für die monoklonalen Proteine gewonnen werden können. Hierfür bieten sich zwei Gründe als Erklärung an:

1. Verglichen mit den Proteinen eines Normalserums, reagieren solche monoklonalen idiotypischen Proteine unterschiedlich mit den Antiseren, weil sie im Vergleich mit dem polyklonalen Spektrum des Normalen eine eingeschränkte Antigenspezifität besitzen. In solchen Seren lassen sich verläßliche quantitative Bestimmungen daher nur für die Nicht-Paraprotein-Immunglobuline durchführen. Die Identifizierung des monoklonalen Proteins erfolgt dagegen mittels Immunelektrophorese. Die mit dem Nephelometer erhaltene relative Konzentrationsangabe für das monoklonale Protein kann höchstens im Einzelfall als Verlaufsparameter herangezogen werden.

2. Bei einigen Patienten mit monoklonalen Paraproteinen (Myelom, M. Waldenström u. a.) sind 7S- und 19S-Globuline der gleichen Proteinspezifität vorhanden. Der Antikörper kann zwischen 7S und 19SProteinen nicht unterscheiden, bei der radialen Immunodiffusion erhält man in diesen Fällen oft Doppelringe.

Wir sind der Ansicht, daß die quantitative Bestimmung einzelner Plasmaproteine, wenn sie mit reproduzierbarer Technik in ausreichender Präzision durchgeführt wird, in absehbarer Zeit die Serumelektrophorese als diagnostische Methode ersetzen kann. Schon heute existieren für zahlreiche Erkrankungen Proteinmuster, die in der Diagnostik benutzt werden können (z. B. Albumin, Transferrin und Komplementproteine bei Nierenerkrankungen; Albumin, Immunglobulin und Transferrin bei entzündlichen Lebererkrankungen; quantitative Bestimmungen verschiedener „akuter"-Phasen-Proteine als Entzündungsparameter, z. B. Haptoglobin oder $\alpha_{1}$ Glykoprotein; Erfassung von Immundefekten mittels quantitativer Bestimmung von Immunglobulinen; quantitative IgM-Bestimmung im Neugeborenenserum als Hinweis auf abgelaufene intrauterine Infektion). Diese Beispiele zeigen, daß quantitative Serumproteinbestimmungen in der klinischen Diagnostik bereits heute einen Platz haben, und daß sie - kritisch angewandt zưr Aktivitätsdiagnose verschiedener Erkrankungsbilder mit herangezogen werden können.

\section{Literatur}

1. Mancini, G., Carbonara, A. O. \& Heremans, J. F. (1963), Immunochemistry 2, 235-254.

2. Mancini, G., Vaerman, J. P., Carbonara, A. O. \& Heremans, J. F. (1964), XI Colloquium on Protides of the Biological Fluids. (Peeters, H. ed.) Amsterdam, Elsevier Publishing Co., p. 370 .

3. Fahey, J. L. \& Mc Kelvey, E. M. (1965), J. Immunol. 94 , 84.

4. Laurell, C.-B. (1966), Biochemistry 15, 45-52.

5. Eckman, I., Robbins, J. B., Van Den Hamer, C. J. A., Lentz, J. \& Scheinberg, I. H. (1970), Clin. Chem. 16, 558-561.

6. Ebeling, H. (1973), diese Z. 11, 209-214. 
7. De Vreker, R. A., De Saussure, V. \& Maxwell, K. W. (1975), Organisation des laboratoires - Biologie Prospective IIIe Colloque de Pont-à-Mousson, 347-351.

8. Hyland, Immunoplate III Radial Immunodiffusion Test for Human IgA, IgG, IgM, Complement C3, Low Level IgA, IgG, and IgM. October 1975.
9. Smith, R.S. (1975), Organisation des laboratoires - Biologie Prospective IIIe Colloque dẹ Pont-à-Mousson, 613-614.

10. Deaton, C. D., Maxwell, K. W., Smith, R. S. \& Creveling, R. L. (1976), Clin. Chem. 22, 1465-1471.
Prof. Dr. med. H. Deicher Abteilựng für klinische Immunologie und Transfusionsmedizin Department für Innere Medizin Medizinische Hochschule Hannover Postfach 610180

D-3000 Hannover 61 